Projets

de paysage

\section{Projets de paysage}

Revue scientifique sur la conception et l'aménagement de l'espace

$22 \mid 2020$

Forêt et paysage

\title{
Ce que les transitions forestières font à l'expérience paysagère
}

Considérations géographiques à partir du cas des robineraies du SudGironde

The Effects of Forest Transitions on the Landscape Experience-Geographical considerations based on the case of the Robinia groves of South Gironde

Sébastien Nageleisen, Baptiste Hautdidier, Laurent Couderchet et Zoé Ginter

\section{OpenEdition}

\section{Journals}

Édition électronique

URL : http://journals.openedition.org/paysage/8637

DOI : 10.4000/paysage.8637

ISSN : 1969-6124

Éditeur :

École nationale supérieure du paysage de Versailles-Marseille, Institut national des sciences appliquées Centre Val de Loire - École de la nature et du paysage, École nationale supérieure d'architecture et de paysage de Bordeaux, École nationale supérieure d'architecture et de paysage de Lille, Agrocampus Angers

Référence électronique

Sébastien Nageleisen, Baptiste Hautdidier, Laurent Couderchet et Zoé Ginter, « Ce que les transitions forestières font à l'expérience paysagère ", Projets de paysage [En ligne], 22 | 2020, mis en ligne le 21 juillet 2020, consulté le 24 juillet 2020. URL : http://journals.openedition.org/paysage/8637 ; DOI : https://doi.org/10.4000/paysage.8637

Ce document a été généré automatiquement le 24 juillet 2020.

Projets de paysage 


\section{Ce que les transitions forestières font à l'expérience paysagère}

Considérations géographiques à partir du cas des robineraies du SudGironde

The Effects of Forest Transitions on the Landscape Experience - Geographical considerations based on the case of the Robinia groves of South Gironde

Sébastien Nageleisen, Baptiste Hautdidier, Laurent Couderchet et Zoé Ginter

1 Lorsque Alexander S. Mather introduit en 1992 la notion de transition forestière, il part d'un constat empirique en attente d'explications théoriques : le retour des forêts dans plusieurs pays industrialisés où elles avaient précédemment fortement régressé. Une littérature dédiée s'est par la suite attachée à documenter la variété des mécanismes ayant pu mener à des patterns régionaux de reconquête des espaces boisés. Si l'exemple français a dans ce cadre été particulièrement commenté (Mather et al., 1999), c'est parce que cette transition est particulièrement nette si on la considère d'un point de vue agrégé, par exemple via l'évolution du couvert national depuis le milieu du XIX siècle. C'est aussi parce qu'elle résulte de la juxtaposition de dynamiques régionales très différentes, articulant ou non une action publique «volontariste " avec des contextes de déprise et de marginalisation des activités agricoles.

2 Trente ans plus tard, une généralisation du processus est observée dans d'autres pays, alimentant des réflexions académiques sur la spécificité et la durabilité de ces mouvements (Sloan et al., 2019), ainsi que l'importance d'une qualification des dimensions discursives et idéologiques de ces processus (Kull, 2017). Le cas français conserve toutefois son intérêt, dans la mesure où l'augmentation des surfaces forestières s'est largement maintenue sur la période: $+2,7$ Mha en France métropolitaine entre 1985 et 2018 (chiffres IGNF, 2019). Or s'il est bien clair que les espaces forestiers regagnés au cours $\mathrm{du} \mathrm{xx}^{\mathrm{e}}$ siècle n'ont pas été des reconstitutions à l'identique, les dynamiques les plus récentes ont également de nombreuses spécificités. Dans le même temps, les regards citoyens sur la forêt ont également considérablement 
évolué, intégrant de nouvelles attentes (bénéfices multiples pour le climat, rôle de refuge, besoin d'usages récréatifs...) et inquiétudes légitimes (sécheresses, méga-feux, ravageurs, statuts des forêts publiques...). Les débats, alimentés par une intense activité éditoriale, portent alors moins sur une crainte de la déforestation que sur la façon dont les trajectoires actuelles de (re)forestation pourraient éventuellement être qualifiées de malforestation. Il importe aussi, pour analyser la diversité des points de vue sur les dynamiques forestières associées à ces transitions, d'en mesurer le caractère prospectif et normatif. Lorsqu'un ou des acteurs attribuent une légitimité à un type de forêt en un instant donné en raison de sa composition spécifique, de sa structure, de sa gestion, ou de ses usages, ils incorporent en effet une logique d'anticipation. Ils intègrent les trajectoires potentielles des formes forestières dans la formation de leur jugement, que ce soit en matière d'attentes, d'espérances ou de rejets (Mansfield et al., 2014). Ces arbitrages peuvent prendre en compte les aspirations à une fréquentation récréative des espaces forestiers et avoir une composante esthétique, laquelle a à voir avec une expérience paysagère qui est souvent peu explicitée.

3 Pour autant, il peut exister un écart important entre besoins ressentis et traduction sur le terrain. Ainsi, cette attention accrue ne se traduit pas par des hausses massives de la fréquentation des espaces forestiers. La relation sensible aux territoires passe beaucoup en Occident par une distanciation de soi à l'espace et une volonté d'artialisation de ce qui est visible, en somme, par le filtre paysager. Cette relation paysagère à l'espace est intégrative et peut être particulièrement bénéfique car elle porte en elle, alternativement ou conjointement, la pratique, le quotidien, le ressenti, la culture. Or si la notion de paysage se prête bien à l'étendue des espaces ouverts de campagnes, de montagnes ou des littoraux, si elle s'est imposée pour les milieux urbains, elle reste à adapter, voire à reconstruire pour des milieux forestiers plus confinés. Pour que le paysage en reste un, nombre d'auteurs semblent en effet considérer la nécessité de maintenir un critère d'ouverture spatiale ou de profondeur visuelle (Balibar, 20181). La forêt est au contraire immersive, intégrative ${ }^{2}$, elle implique une confrontation à des attributs de la fermeture. Est-ce alors du paysage au sens où nous venons de l'évoquer?

4 Nous proposons ici de rendre compte de la complexité de ces rapports aux paysages résultant de transitions assez largement impensées de l'action publique. Nous nous appuyons sur l'exemple des espaces boisés dominés par le robinier faux-acacia, une essence nord-américaine introduite en France au XVII siècle. L'ambiguïté du statut de cet arbre nous permet d'articuler à la notion de mal-forestation celle de malpaysagement. Initialement associée à des usages d'ornement puis essentiellement agricoles et viticoles, l'espèce est passée par plusieurs cycles de valorisation et d'oubli relatif. Elle se distingue actuellement par une expansion, qui pour être diffuse, à bas bruit et selon des relations privilégiées avec les infrastructures linéaires, n'en est pas moins rapide et spectaculaire. Ces dynamiques mènent à des appréciations contradictoires des paysages associés à «l'acacia». Qualifié par certains de peste végétale invasive, de concurrent féroce de la biodiversité autochtone, voire d'emblème de la malforestation en raison de sa capacité de colonisation des espaces ouverts, il est défendu par des habitants et une partie des acteurs forestiers comme un auxiliaire des pratiques paysannes aux fleurs odorantes et mellifères, un arbre lumineux et peu exigeant pour les jardins, un bois dont les qualités d'imputrescibilité et le pouvoir calorifique devraient justifier un plus large usage, tant domestique qu'industriel. Le parallèle entre les registres forestier et paysager est ici flagrant, l'acacia réveillant une opposition théorisée par Yves Luginbühl (1989) entre paysage élitaire et paysage 
ordinaire. Envahissant et peu sociable, consommateur récent d'espaces ouverts, espèce de lisière, est-il seulement forestier?

Nous posons la double hypothèse de sa sous-évaluation dans les inventaires forestiers (puisqu'il est tenu à l'écart de la sylviculture et des politiques forestières, élitaires en quelque sorte) et de sa surévaluation dans le paysage ordinaire (en raison de la place qu'occupe l'arbre dans l'espace visible). Même si sur notre terrain d'étude, il finit par constituer des massifs conséquents, l'appréhension paysagère que nous en faisons nous place plus à distance ou en dehors qu'en immersion.

Pour répondre à la première partie de notre hypothèse, nous complétons la cartographie forestière par une classification d'images satellites localisée, destinée à préciser l'existence de décalages entre bases institutionnelles et la réalité. La seconde partie de notre hypothèse est traitée au moyen d'un échantillonnage photographique réalisé depuis les chemins et d'une modélisation SIG. L'analyse nous permet de quantifier la place du robinier parmi les arbres associés à l'image de la composition forestière vue de l'extérieur de la forêt. Ce croisement d'approches offre une lecture plus fine des modalités de ces transitions forestières. Un recours complémentaire à des sources qualitatives (entretiens et archives) ouvre ensuite des pistes de réflexion quant aux trajectoires et représentations associées des divers paysages mis en évidence.

\section{Terrain et méthodes}

\section{Le Sauternais : une région viticole marquée par plusieurs vagues de transitions forestières}

7 Le croisement d'approches est mis à l'épreuve sur une zone d'étude relativement circonscrite, le Sauternais. À $40 \mathrm{~km}$ en amont de Bordeaux, sur des terres alluvionnaires de la rive gauche de la Garonne, cette zone est traversée selon un axe nord-sud par la vallée du Ciron. Avec ses affluents, le Tursan et l'Arec (ruisseau de la Mouliasse), cet espace alluvial abrite des surfaces forestières feuillues notables: chênaies, frênaies, aulnaies. La très ancienne présence de l'activité viticole, pour l'essentiel en appellation Sauternes à l'est du Ciron ou Graves à l'ouest (Budos et Landiras), a été à l'origine du développement de boisements dédiés à l'équipement de la vigne, échalas puis piquets. Ce rôle, initialement dévolu au châtaignier, a été repris à partir du XVIII ${ }^{e}$ siècle par le robinier faux-acacia, dont les superficies ont ensuite grimpé très nettement à partir de la fin du XIX ${ }^{e}$. Au sud de la zone, l'influence du massif landais est présente, tant dans la nature des sols (sableux) que par la dominance du pin maritime et de son mode de gestion intensif (plantations équiennes monospécifiques exploitées à trente ans). Nous choisissons de centrer l'essentiel de nos analyses sur un rectangle de 8700 ha d'orientation est-ouest $(13,5 \times 6,5 \mathrm{~km})$, qui présente cette diversité des contextes paysagers et des dynamiques du couvert arboré qui leur sont associées (figure 1). 
Figure 1. Carte de localisation de la zone d'étude

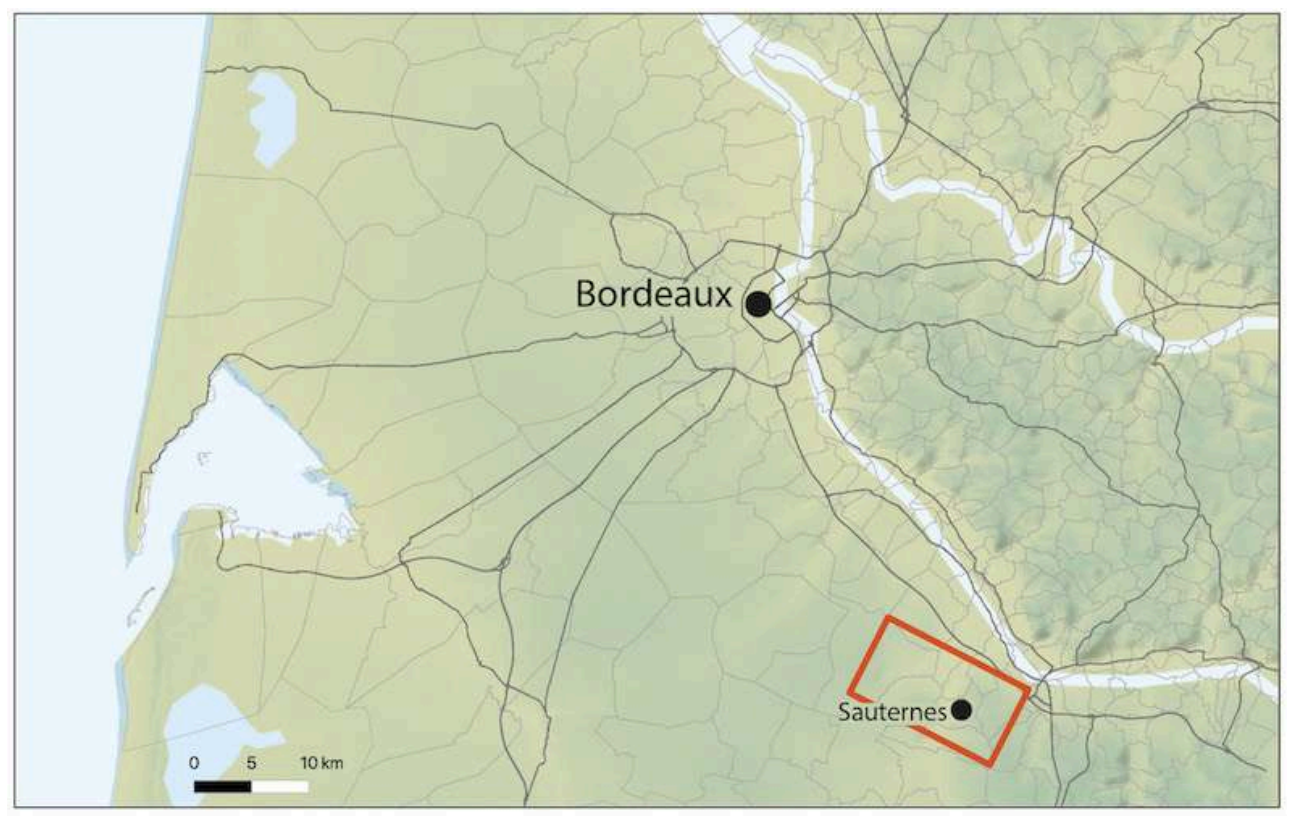

Sources : auteurs, BD Alti $\AA$ et BD Carto $\AA 2020$

\section{Par le haut : une classification ad hoc à comparer avec les cartes officielles}

Un produit cartographique phare de l'inventaire forestier national, la base BD Forêt ${ }^{\oplus}$, fournit des informations très détaillées sur la nature et les évolutions des espaces dits «naturels et semi-naturels» de la France métropolitaine. Depuis sa version 2.0, produite à partir de 2007 (avec un passage en 2014 pour le département de la Gironde), elle renseigne une trentaine de "types de formations végétales ", allant, lorsque c'est possible, jusqu'à la discrimination de 16 essences forestières (IGN, 2016). Par construction, les critères de géométrie des objets cartographiés ( 0,5 ha de superficie et $20 \mathrm{~m}$ de largeur) laissent de côté une part potentiellement non négligeable de la dynamique actuelle des couverts arborés : boqueteaux, lisières et emprises linéaires, composition des mélanges.

La mise à disposition massive de données satellitaires optiques à haute résolution a été présentée comme l'opportunité d'une réponse à ce manque. Chaque image satellite traduit un instantané de l'état du milieu. Lorsque les données sont disponibles, la multiplication de ces instantanés permet de considérer la dynamique de végétation comme critère de reconnaissance des couverts végétaux. Les classifications ne considèrent plus alors des points dans un espace multidimensionnel mais des vecteurs (Couderchet, 2008). La phénologie des paysages forestiers étant en effet marquée par des décalages entre espèces, dans les événements de feuillaison, floraison ou de jaunissement automnal, le signal électromagnétique enregistré par les satellites d'observation de la terre sur un seul cycle annuel peut saisir cette complexité de la dynamique des paysages. Permise par une amélioration des fréquences de revisite et des résolutions spatiales, la reconnaissance des peuplements sur la base d'exploitations numériques de séries temporelles est une réalité opérationnelle récente. Reprenant les propositions de Nicolas Karasiak et al. (2017), nous utilisons 12 images composites ${ }^{3}$ 
couvrant la totalité du déroulement de l'année 2018 pour construire une classification supervisée des couverts forestiers de la zone d'étude. La résolution spatiale de notre carte est celle des images que nous utilisons, chaque pixel mesurant $10 \mathrm{~m}$ de côtét .

Par des méthodes simples de télédétection (création de matrices de confusion, calcul de statistiques d'écologie du paysage), nous pouvons alors juger de la qualité de notre classification et établir des points de comparaison avec la cartographie institutionnelle de la BD forêt.

\section{Par le bas : part et composition des lisières boisées dans une analyse paysagère systématique}

11 Le paysage fait appel à la géométrie de la tangente et non à celle de la projection. La géométrie de la profondeur retenue, avec ses espaces ouverts et ses espaces masqués, est beaucoup plus compliquée à appréhender que la géométrie classique de la projection. Les échelles se dilatent dans les plans rapprochés et se contractent dans les plans éloignés, l'espace représenté ne traduit plus l'exhaustivité de la carte. L'ambition de confrontation des reconnaissances d'une espèce végétale par la carte et depuis un itinéraire relève sur ce registre de la gageure, les topologies de la carte et du paysage ne coïncidant pas. L'objectif de notre démarche n'est donc pas de confirmer un regard par l'autre mais au contraire de mesurer en quoi l'entrée institutionnelle, celle de la BD forêt ou celle qui passe par l'exploitation des images satellite, peut produire - sur l'importance d'un élément donné du paysage -des représentations différentes de celles qui sont produites par le canal à l'origine des connaissances vernaculaires.

Notre approche par le paysage mobilise l'enquête photographique et la modélisation SIG. Les deux méthodes sont complémentaires. L'approche photographique de terrain est déterminante car elle procure aussi une compétence spécifique traduite ensuite dans le paramétrage de la modélisation: jusqu'à quelle distance reconnait-on une lisière ou une haie? Distingue-t-on un robinier d'un autre feuillu? L'approche modélisatrice SIG réalisée à la suite de l'enquête photographique peut être paramétrée en fonction de l'expérience acquise, elle permet d'obtenir des résultats similaires mais provenant de techniques et de sources très différentes. Un deuxième jeu de résultats est donc obtenu permettant une comparaison particulièrement riche d'informations.

Notre approche par le paysage s'effectue depuis un itinéraire (Griselin et Nageleisen, 2004 ; Nageleisen, 2007) sélectionné pour la diversité des espaces traversés et pour sa représentativité. Il s'agit de se mettre en situation et de parcourir les lieux afin d'en saisir de manière systématique les paysages visibles. Ainsi 42 kilomètres ont été parcourus et échantillonnés en réalisant tous les kilomètres deux panoramiques de $180^{\circ}$ afin d'obtenir une information à $360^{\circ}$. Chaque image est renseignée avec sa position géographique et les pourcentages des éléments qui la composent. Cette étape permet d'aborder finement la composition paysagère des espaces traversés. La part du ciel, des forêts ou des robiniers dans chaque cliché est par exemple calculée précisément. Quantifier le paysage de cette manière peut sembler bien éloigné de l'expérience paysagère vécue par celui qui fréquente les lieux. En travaillant sur ces photographies nous n'avons pas l'ambition de reproduire une expérience paysagère complète mais plutôt d'évaluer par un échantillonnage ce que la vue tangentielle implique dans nos éventuelles représentations des transitions forestières. La modélisation sous SIG met en œuvre une carte numérique du relief à cinq mètres de 
résolution (IGN), elle prend en compte la hauteur des bâtiments et de la végétation à partir des informations de la BD Topo (IGN) et de la couche d'occupation du sol réalisée pour l'approche par le haut. Les calculs de visibilité sont effectués pour chaque lieu photographié et prennent en compte le cadrage des clichés (hauteur et largeur). Le tout est réalisé avec le logiciel Pixscape (Sahraoui et al., 2016).

Figure 2. Budos, abords du château Duverger, une vue semi-panoramique (a) et sa modélisation sous Pixscape (b)

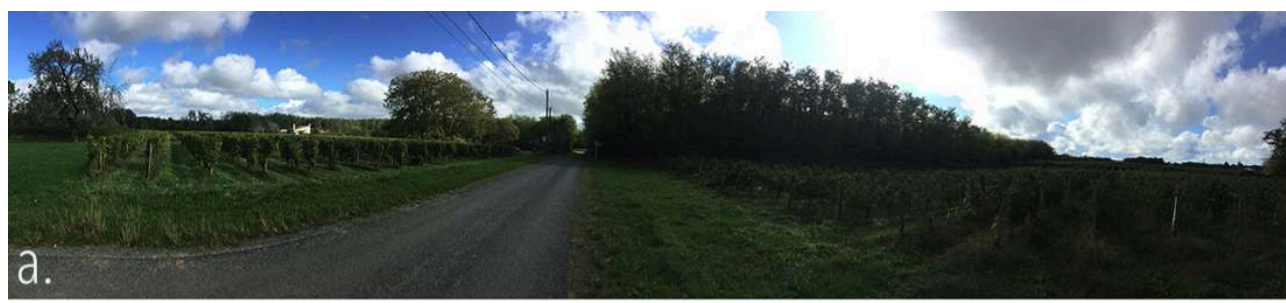

b.

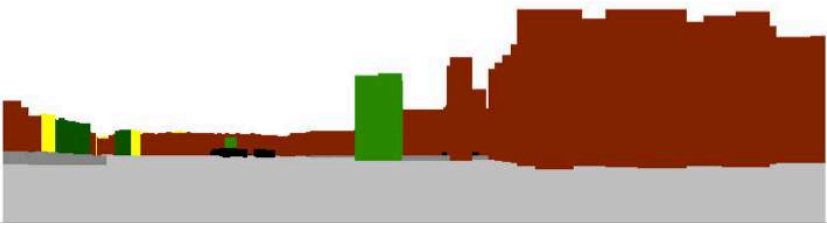

Source : auteurs, 2020.

14 Les différences entre les résultats obtenus par les deux méthodes permettent d'affiner la modélisation sous SIG qui pourra ensuite être généralisée à l'ensemble de l'espace étudié. Cette tâche a ici pu être testée avec un semis de 1544 points, éloignés de plus de $50 \mathrm{~m}$, échantillonnés sur l'ensemble de l'emprise du réseau routier de la BD Topo. L'objectif du test est moins la recherche d'une exhaustivité quantitative ou de validation que la mise en perspective des résultats saillants obtenus sur les étapes du parcours de l'enquête photographique. 
Figure 3. Situation de la zone d'étude, classification du couvert arboré

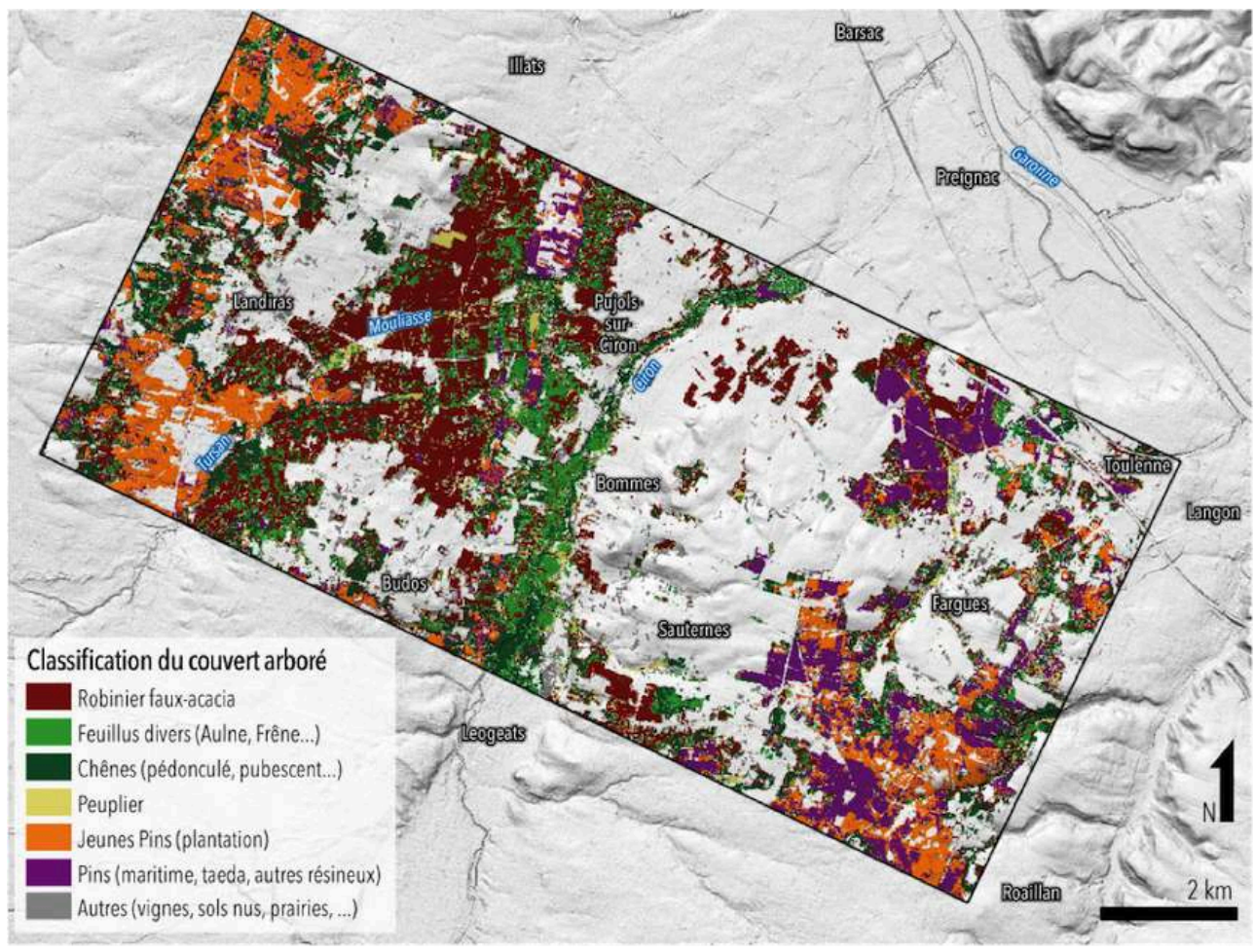

Source : auteurs, 2020.

\section{Une présence diffuse, des modalités contrastées}

Les deux matériaux empiriques principaux (photographie et classification automatisée d'images satellite) offrent comme attendu une lecture plus fine que la seule source institutionnelle, au-delà du constat convergent d'une place importante des boisements feuillus dans les paysages. Si dans ce tableau dominent les taillis de robinier, les modalités de leurs arrangements spatiaux en sont toutefois assez différenciées.

\section{Par le haut : la réévaluation produit d'abord une redistribution spatiale}

Les tableaux de la figure 4 croisent la typologie des couverts forestiers produite par classification d'images satellite ${ }^{5}$ avec les vérifications de terrain et les distinctions d'essences opérées dans la cartographie standard de la BD Forêt v2 ${ }^{6}$. 
Figure 4. Matrices de confusion de la classification avec les points de validation (a) et la cartographie institutionnelle (b), 2014

a. Avec les données de validation (nombre de points)
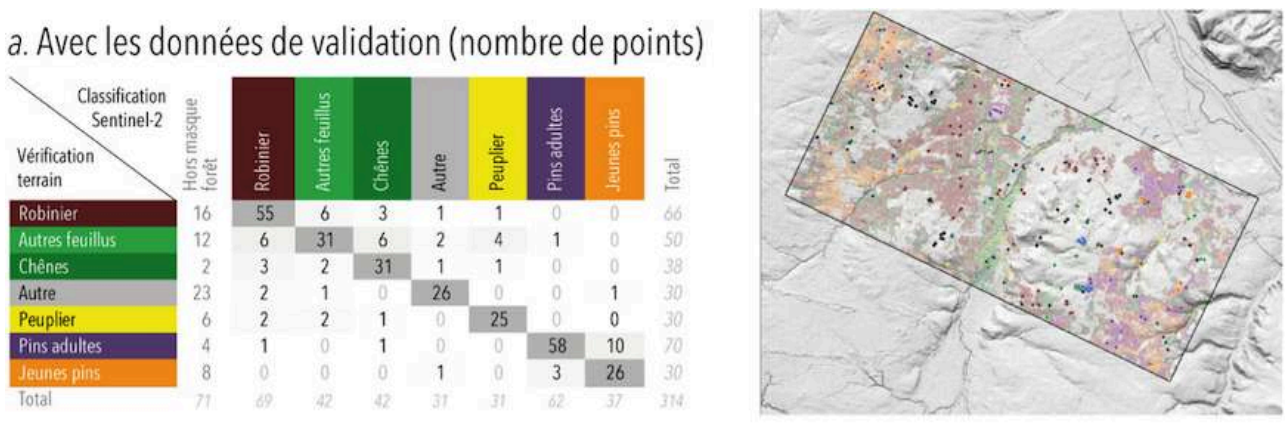

b. Avec la cartographie de l'inventaire forestier (en ha)
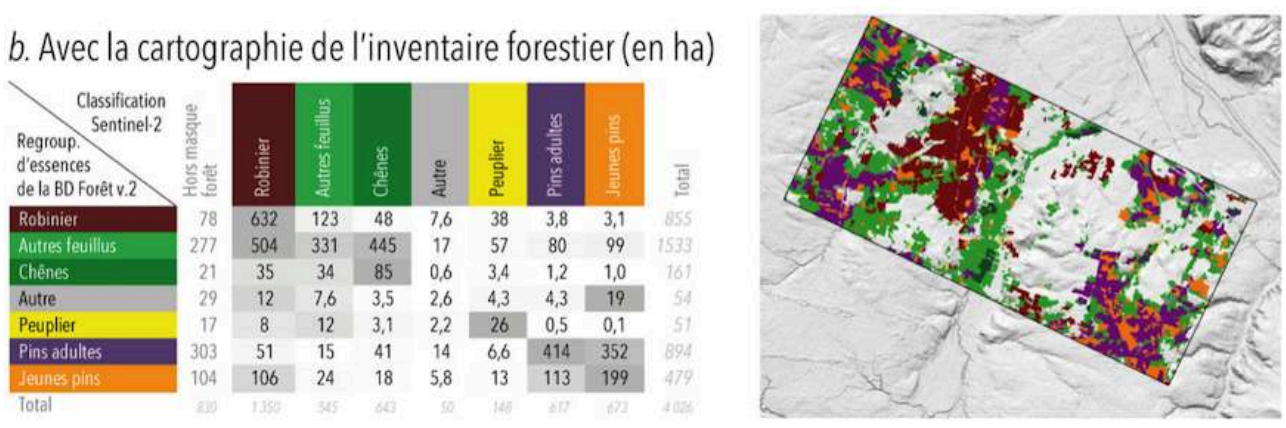

Source : auteurs, 2020.

17 On observe ainsi un agrément satisfaisant dans le premier $\operatorname{cas}^{7}$, l'essentiel des confusions se situant aux interfaces de la catégorie «feuillus divers » et de celles des robiniers et chênes, ainsi que sur l'identification de deux stades de développement de la pinède. L'articulation avec la cartographie de «l'inventaire forestier » repose sur une base beaucoup plus fragile ${ }^{8}$ : les confusions sont opérées sur les mêmes catégories, mais dans des proportions plus importantes. On note par ailleurs la contrainte d'un effet millésime imposé par la disponibilité de nos produits: la base IGN est en effet antérieure de quatre ans à notre classification (été 2014). Si ce délai est a priori court pour saisir d'éventuelles dynamiques structurelles, il importe ici de considérer le contexte forestier régional. Une tension généralisée sur la ressource bois est en effet à l'œuvre, sous la conjonction des dégâts massifs de la tempête Klaus de 2009 et de l'essor de la demande pour le bois-énergie (Hautdidier et al., 2018; Banos et Déhez, 2017). L'ampleur des transitions observées peut être en partie expliquée par ce double mouvement : reconstitution de parcelles impactées, avancement d'un passage en coupe pour répondre aux trous de production du marché régional. Ces évolutions récentes, si elles étaient univoques, devraient avoir tendance à aller dans le seul sens du modèle intensif dominant, celui du pin maritime. Or, ce n'est pas le cas à l'échelle de notre zone d'étude. Les espaces pour lesquels la progression est la plus nette sont ceux identifiés en robineraies : + 57 \% par rapport à celles de la BD Forêt, principalement établies aux dépens de la classe générique « feuillus divers ».

Si la classification proposée donne de bonnes raisons de penser qu'une essence comme le robinier serait potentiellement sous-estimée par la cartographie institutionnelle, le chiffrage de ces superficies importe ici moins que les modalités du gain. À ce jeu du 
comment plutôt que du combien, on identifie ainsi trois mécanismes qui expliquent l'essentiel du décalage.

1. le traitement du mélange : le gain de résolution maintient un agrément apparent des deux cartographies sur des espaces en peuplements purs mais permet aussi une analyse très différente des parcelles dans lesquelles le mélange d'essences est manifeste ;

2. les disparités des conditions d'accessibilité aux terrains: les espaces les moins aisément prospectables et validables ont tendance à être affectés aux classes les plus génériques de la cartographie institutionnelle (une part importante de la vallée du Tursan, qui dans notre cas bascule fortement $\mathrm{du}$ " feuillus divers » au robinier, a une faible accessibilité routière);

3. la prise en compte des objets linéaires, empêchée par construction dans la BD forêt, fait ici apparaître les spécificités de la composition des lisières et des peuplements associés aux infrastructures linéaires, abords de route et délaissés.

\section{Par le bas : être visible sans pour autant être repérable?}

L'enquête photo permet de constater la présence de robiniers sur $58 \%$ des clichés et il représente plus de $10 \%$ de la scène paysagère dans $60 \%$ des cas. Les $40 \%$ restant correspondent donc à une présence plutôt ponctuelle, à des jeunes plants, ou à une vue à distance des peuplements. Le robinier est évidemment très lié à la présence de forêts puisque, dans ce milieu, sa présence est de $78 \%$. Le potentiel d'analyse qu'offrent les photographies est très important mais nous en limitons l'usage, dans le cadre de cet article, au paramétrage des calculs de visibilités généralisables ensuite à l'ensemble de l'espace étudié. La figure 5 montre que les résultats obtenus sont globalement proches du recensement sur le thème général des forêts, la concordance étant moins nette pour le comptage des robiniers. Dans ce cas, on relève une surévaluation de la modélisation SIG par rapport à l'analyse photographique. Ce constat s'explique évidemment par la difficulté de reconnaître une espèce végétale à partir des photographies dès qu'elle se situe à distance, alors que l'analyse mobilisant une base de données thématique n'est pas limitée par cet effet de profondeur des vues.

Figure 5. Comparaison des résultats des visibilités modélisées avec l'analyse visuelle des clichés

Pourcentage des forêts dans la scène paysagère

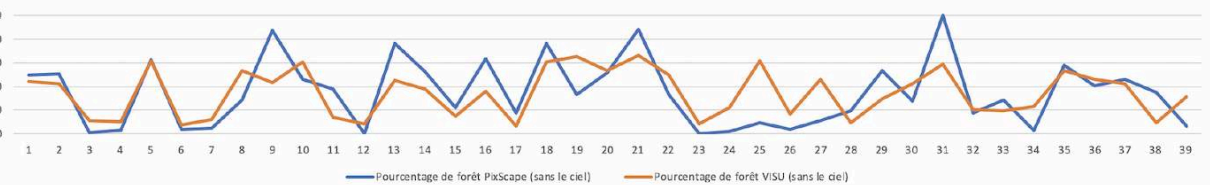

Pourcentage des robiniers dans les éléments forestiers de la scène paysagère

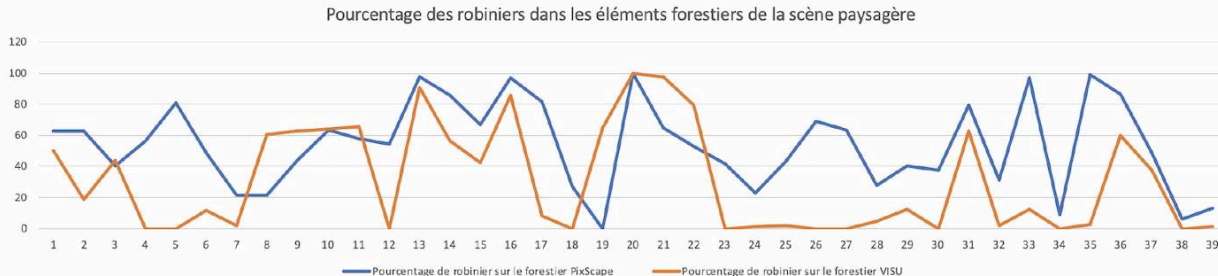

Source : auteurs, 2020.

Projets de paysage, 22 | 2020 
La figure 6 permet de visualiser le lien entre modélisation et analyse visuelle pour quatre métriques. La surévaluation est la plus nette pour les vues modélisées de l'acacia, bien que le nuage de points présente deux groupes assez clairement disjoints. Outre les erreurs de la classification, ce décalage s'explique par deux facteurs:1) de près, l'analyse visuelle des lisières traduit bien la présence de mélange, notamment avec des essences de sous-étage qui restent peu accessibles à la vue zénithale ;2) de loin, la modélisation a des certitudes que l'analyse visuelle des clichés ne peut atteindre.

Figure 6 : Comparaison des valeurs mesurées et modélisées pour quatre métriques en vue tangentielle

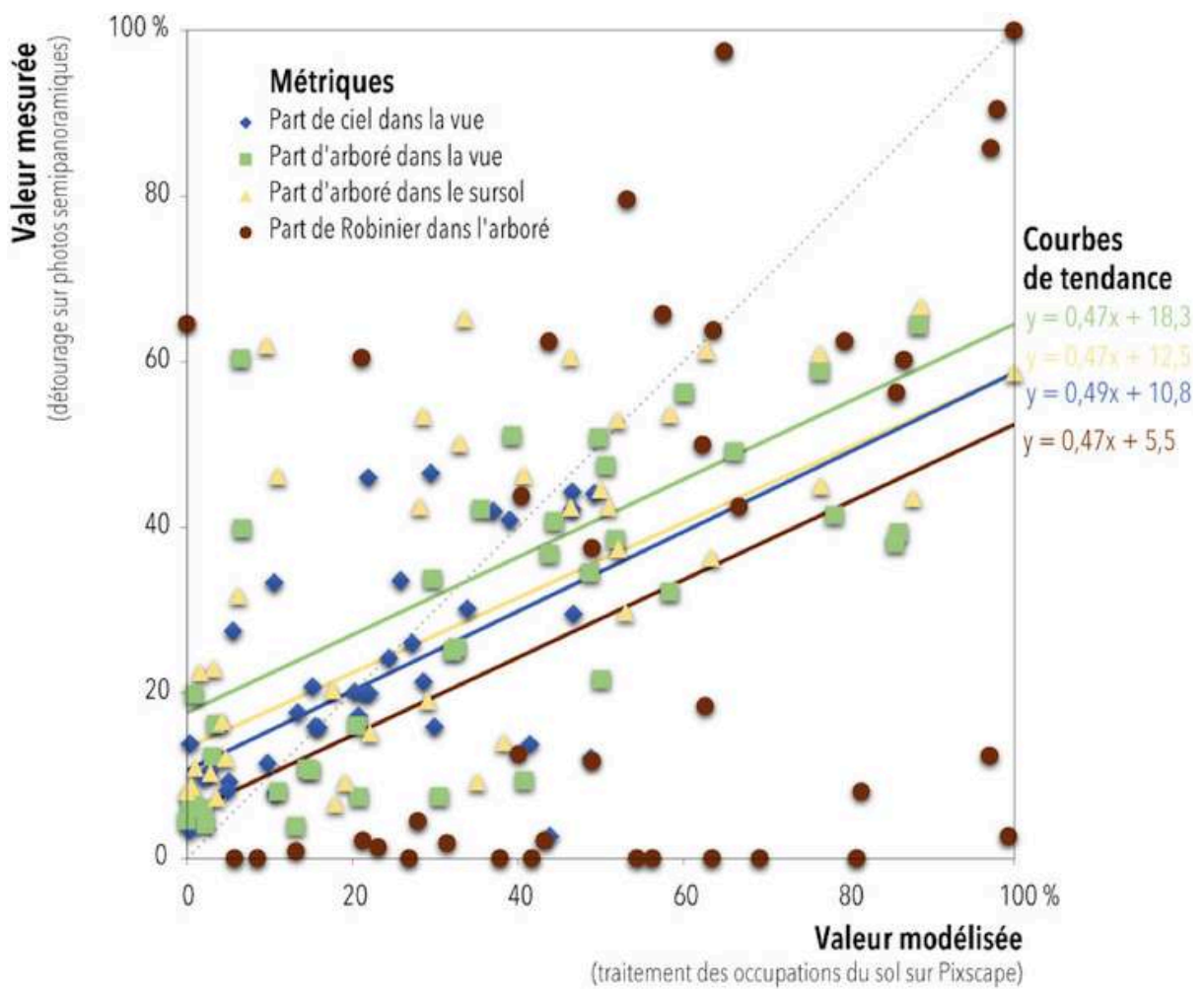

Source : auteurs, 2020.

L'extension de la modélisation des vues tangentielles à l'ensemble de la zone d'étude pourrait donner lieu à une typologie et à une analyse qui dépasseraient le propos de l'article. Il est néanmoins intéressant, pour recadrer nos interprétations, de présenter quelques résultats obtenus depuis les 1544 points de notre échantillon. Cette multitude de points tests montre que seuls $2,4 \%$ des scènes paysagères modélisées ne comptent pas d'acacia dans leur composition. Les calculs ont été réalisés avec une distance maximale de $2 \mathrm{~km}$, soit un ordre de grandeur bien supérieur à celui permettant une identification visuelle des espèces en lisière. Un croisement de la modélisation et de la classification interprété en ces termes aurait tendance à appuyer le constat d'une omniprésence de l'espèce dans les paysages visibles des axes routiers du Sauternais, bien qu'elle ne soit pas forcément repérable, même pour un observateur attentif. Audelà des limites visuelles évidentes, nous expliquons aussi cette sous-évaluation des robiniers par l'impact du déplacement de l'observateur. En effet la cinétique paysagère induite révélerait les essences majoritaires des massifs boisés, le déplacement de 
l'observateur, surtout s'il est rapide, impliquant une simplification de l'information paysagère perçue. Malgré ces biais, l'approche suggère également que les situations où l'arbre aurait l'exclusive sont tout aussi rares. En effet, seuls $5 \%$ des points se caractérisent ainsi par une part de l'acacia supérieure à $95 \%$ du couvert arboré visible, ce chiffre baissant à $0,8 \%$ pour une part de $100 \%$. Toutes conquérantes qu'elles puissent être, les insertions paysagères de l'arbre se font sur un mode plus "partagé » qu'il pourrait paraître au premier abord.

\section{L'acacia dans les paysages: une présence substantielle mais composite}

Il ressort également que les dynamiques du robinier faux acacia se déploient dans le Sauternais selon une grande diversité de contextes paysagers. D'une présence très dominante, au sein d'un massif continu à l'ouest, aux plantations en "timbre-poste » au sein de la matrice viticole, les occurrences sont plus ou moins marquées sur l'ensemble de la zone d'étude. Cette hétérogénéité s'explique en partie par des pratiques et histoires paysagères variées. Plusieurs configurations peuvent être mises en évidence.

\section{La forêt de production habitée}

La configuration la plus visible est celle de la forêt de production qui fait l'objet d'une reconnaissance paysagère ancienne. À l'ouest, le «massif » de la vallée de la Mouliasse est la plus grande robineraie d'un seul tenant de France. Bien que la propriété y soit très morcelée, la densité du peuplement offre un paysage fermé semi-perméable au cœur duquel les ouvertures sont résiduelles (hameaux, cultures...). Il s'agit d'une forêt de production, activement exploitée depuis la fin du XIx ${ }^{e}$ siècle. À côté de ce massif, des boisements plus dispersés témoignent de l'association historique de l'espèce aux pratiques agricoles, et surtout viticoles. Le robinier trouve sa place sous la forme d'îlots ${ }^{9}$ au cœur d'une composition de vignes et de forêts feuillues usagères de dimensions plus modestes. À l'échelle du paysage, les transitions entre la forêt fermée et des horizons moins denses, avec l'ouverture des vignes et des prairies, sont communes. 
Figure 7. Boisements de production
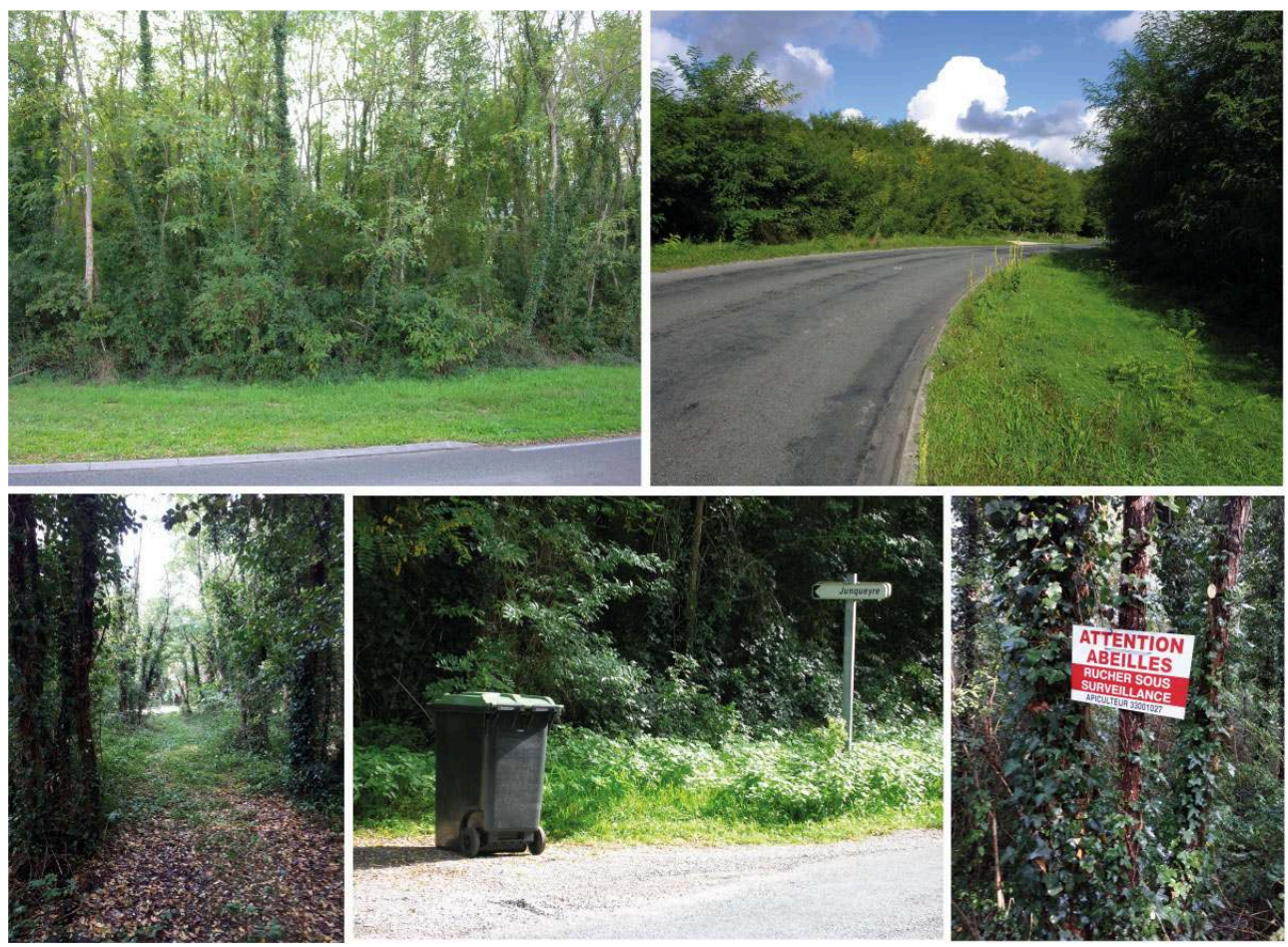

Source : auteurs, 2020.

\section{Les peuplements « spontanés »}

D'autres formes de boisements plus fortuits offrent une présence paysagère plus diffuse et accidentelle, mais assez largement répandue. Du fait de sa croissance rapide et de son caractère pionnier, le robinier occupe facilement les lisières forestières, quitte à donner l'impression d'un peuplement monospécifique. Les vagues d'abandon des surfaces viticoles (avec la crise du phylloxéra et les phases de surproduction) ont vu les modalités de présence du robinier se diversifier. Ces dynamiques ont contribué à la modification du paysage et à la présence actuelle de boisements spontanés. Cette conquête des friches ou encore des bords d'infrastructures linéaires augmente la place de l'arbre dans le paysage tout en le reléguant à un statut marginal, parfois localement accentué par une proximité spatiale avec les abords de hameaux où l'habitat pavillonnaire a pu récemment se développer. 
Figure 8. Boisements spontanés

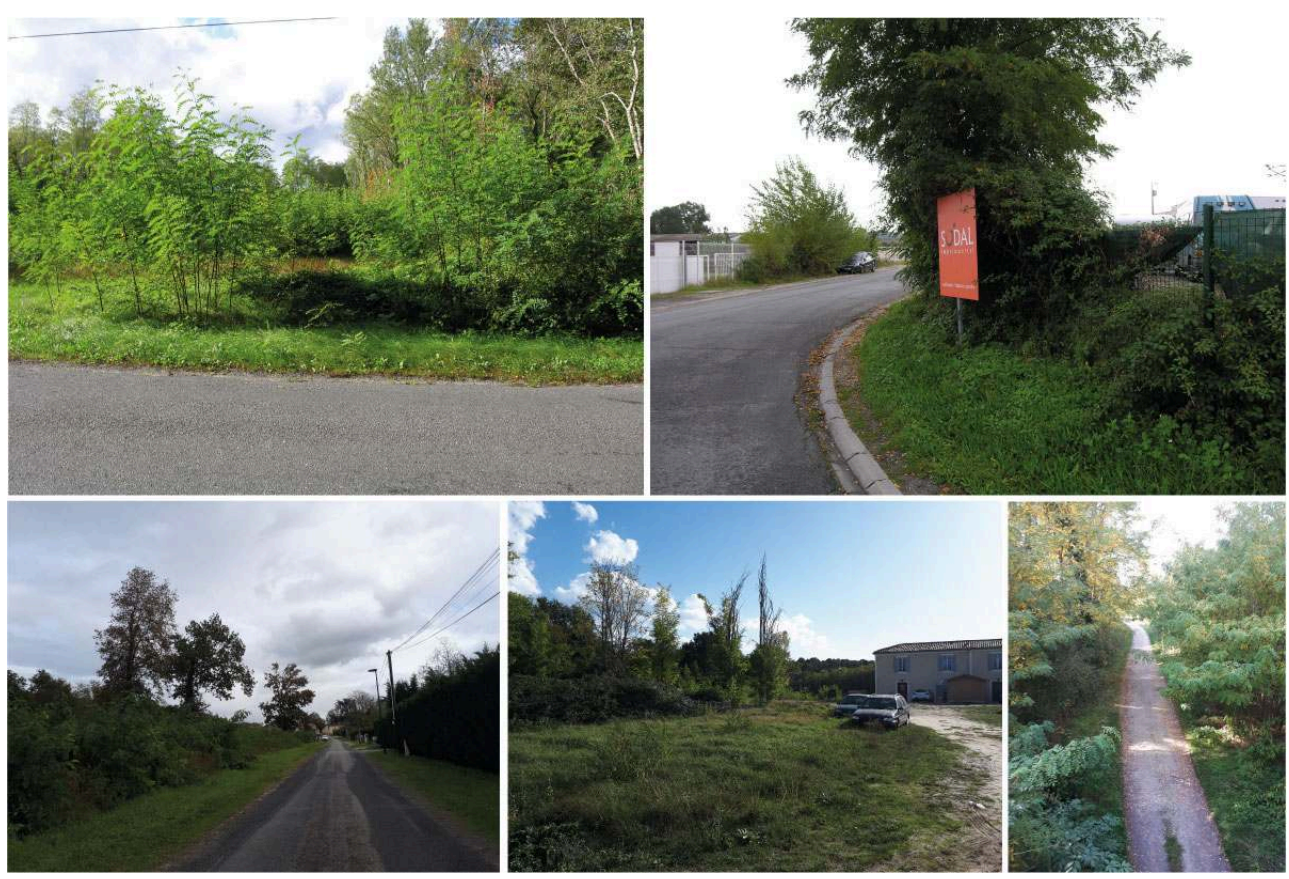

Source : auteurs, 2020.

\section{La présence ornementale}

Motif premier de l'introduction de l'espèce, l'acacia est encore assez largement présent comme arbre d'ornement. Dans notre zone d'étude, il s'insère dans les jardins, le long des avenues ou encore devant les mairies. Le nombre de rues et de places nommées «acacia» n'est d'ailleurs pas anodin. Apprécié pour sa floraison et sa présence ornementale, l'acacia s'installe en petits bosquets au cœur des hameaux ou encore au sein des bois marmenteaux, peuplements d'agrément situés en contiguïté des châteaux. 
Figure 9. Boisements ornementaux
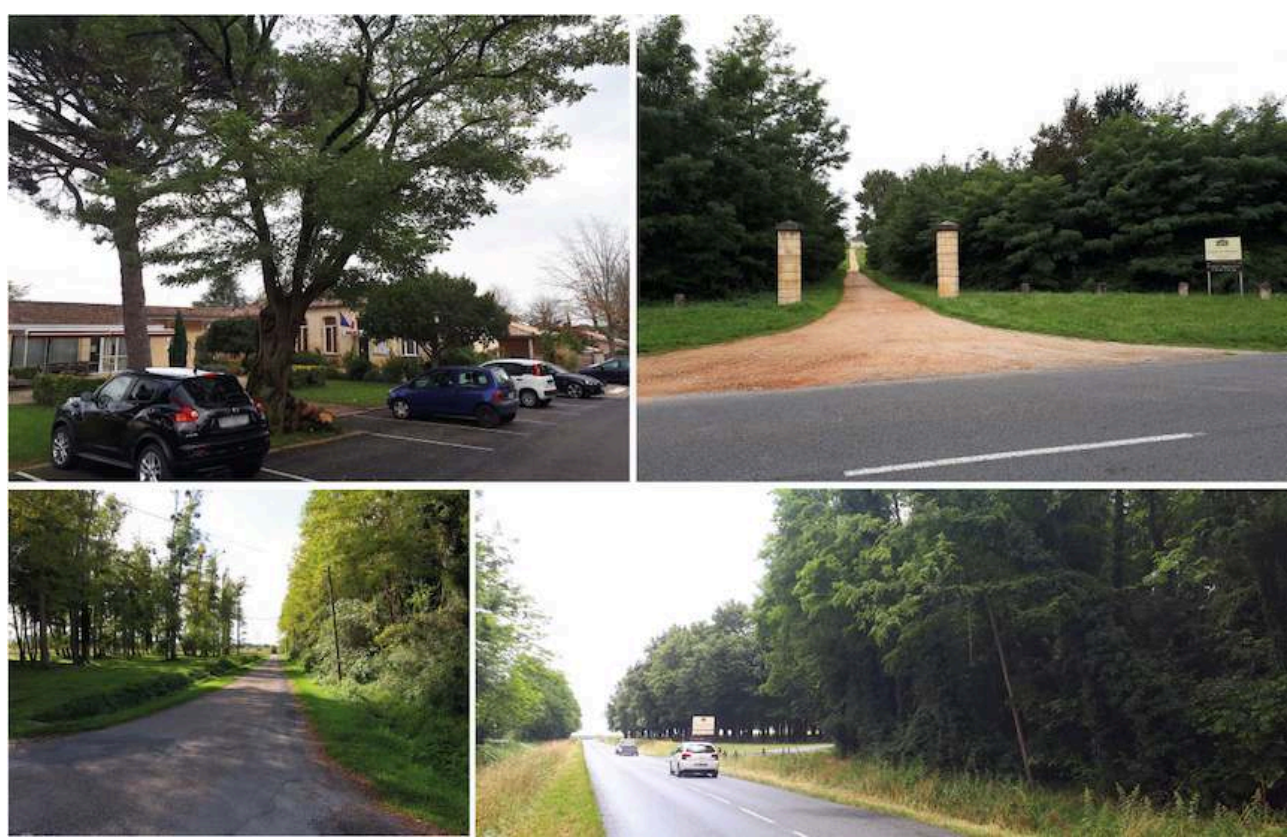

Source : auteurs, 2020.

Cette présence paysagère, le plus souvent revendiquée et lisible, peut également avoir une dimension accidentelle, pour laquelle il est difficile d'évaluer le degré d'opportunisme des propriétaires et gestionnaires, dans ce qu'il serait tentant d'interpréter comme un accompagnement, voire une promotion de l'arbre. Ainsi, les bois marmenteaux encadrant l'entrée du domaine de Rabaud-Promis (figure 9, en haut à droite) ont été constitués par un peuplement quasi pur de robiniers qui a été essentiellement installé en reconstitution des dégâts massifs de la tempête de décembre $1999^{10}$. La situation est différente sur l'entrée nord du château Suduiraut (figure 9 , en bas à droite) : les parcelles des bois marmenteaux bordant le chemin d'accès sont en grande majorité de la chênaie, débouchant sur une allée de pins francs (parasol). Mais des accrus d'acacias sont fortement présents et clairement gardés comme tels sur une longueur importante de lisière, dans une continuité avec des parcelles voisines, qui elles sont exclusivement du taillis destiné à la production de piquets. Dans un plan du domaine datant de $1848^{11}$, les deux parcelles de ce que l'on pourrait actuellement qualifier de chênaie "mixte» sont alors affichées comme contenant des pins et des acacias. L'ancienneté d'une promotion explicite du robinier est ici manifeste - et permet donc d'imaginer qu'il existe là une certaine expérience sur les objectifs et les modalités d'une gestion de l'essence. Pour autant, cela n'implique pas l'abandon d'une forme de hiérarchie dans l'appréhension des qualités paysagères et patrimoniales des essences forestières. Accepté, utilisé, visuellement intégré, l'acacia n'en a pas moins été «contenu » au profit d'une autre essence dont on peut penser qu'elle a été jugée plus «noble». 


\section{Visibilités paysagères, invisibilités institutionnelles: les reconnaissances paradoxales d'une transition à bas bruit}

La diversité des contextes paysagers et des dynamiques du couvert arboré qui leur sont associées, caractérise aussi l'ambiguïté des espaces boisés. Les robineraies apparaissent comme une composante essentielle, mais hétéroclite, des paysages de la zone d'étude. Pour autant, une enquête qualitative complémentaire et des entretiens semi-directifs menés auprès d'acteurs (forestiers, institutionnels, habitants) dans la région révèlent que l'espèce ne fait pas l'objet d'une reconnaissance institutionnelle marquée, ni homogène.

Les représentations et appréhensions de ces espaces sont plurielles. Les acteurs forestiers reconnaissent leur omniprésence («ils sont à peu près partout », entretien, 2019) et cherchent à en tirer profit, ils font également volontiers allusion à l'esthétisme des peuplements cathédrales. Pour les habitants et usagers, leur présence est ancrée dans les représentations, témoignant d'une appropriation sur le temps long. En parallèle, les acteurs naturalistes regrettent une banalisation du paysage, notamment dans la vallée du Ciron, attribuée aux boisements spontanés de robiniers. Cette visibilité paysagère, si elle est reconnue, n'est pas le support actif d'une stratégie de valorisation ou de patrimonialisation. Au niveau des documents d'aménagement et d'urbanisme des communes concernées, les habitats et milieux naturels sont aisément associés aux taillis de robinier. Pourtant, les formes plus spontanées et impensées de ces robineraies sont assez largement invisibilisées par l'action publique. La documentation des sites inscrits ou classés (Sauternais, Yquem, Suduiraut) exprime cet entre-deux : le lien historique entre la viticulture et l'acacia n'y est pas caché, mais l'acceptation de la présence de l'arbre n'y vaut pour autant pas patrimonialisation auprès des acteurs interrogés. On observe donc des reconnaissances paysagères paradoxales, entre l'impensé et la valorisation à bas bruit.

\section{Conclusion}

La mise en place conjointe des diverses méthodes présentées dans cet article permet d'appréhender finement les modalités de transitions forestières peu saisies par les moyens traditionnels de la cartographie. Ces transitions sont aussi évidemment repérées et recensées sur le terrain par les professionnels. Leur généralisation et leur accentuation, sous l'effet conjoint du réchauffement climatique et des changements de politique actuels, vont néanmoins rendre plus difficile leur évaluation précise, exhaustive et objective. L'expérience menée dans le Sauternais montre que l'entrée paysagère est une solution pour mieux appréhender de manière systématique ces changements importants. L'usage des photographies, bien que long à mettre en œuvre, paraît être une étape essentielle pour paramétrer les calculs de visibilité que l'on pourra utiliser ensuite de manière plus généralisée. La méthode s'attache à maintenir dans un même modèle spatial théorique les vues paysagères et cartographiques pour une meilleure compréhension de la place de l'arbre dans les représentations. d'enseignements. En parcourant les lieux, il apparaît clairement que les robineraies 
incarnent un contrepoint aux formes régionales de l'«idéal paysager forestier ». Les taillis destinés à la production de piquets, dont les ambiances sont a priori peu accueillantes, émergent comme une figure centrale de ce tableau. Ils cohabitent étroitement avec des boisements spontanés largement impensés, ne faisant souvent l'objet d'une appropriation par l'usage qu'à la période de floraison, ainsi qu'avec les formes de valorisation paysagère beaucoup plus explicites des bois marmenteaux de domaines viticoles. Il existe donc un paradoxe de la diversité des possibles expériences paysagères envers ces boisements: parfois superficielles et peu articulées, symptomatiques d'un «mal-paysagement ", elles sont le plus souvent compatibles avec une invisibilisation par les instances publiques... Semblant n'inciter qu'à une fréquentation superficielle et fugace, elles n'en sont pas moins le support - suivant en cela Becky Mansfield et al. (2014) - d'aspirations à l'intimité et à la sécession qui ne recoupent pas nécessairement les logiques naturalistes, récréatives ou "filières " auxquelles on serait tenté de les réduire. Ces expériences pourraient alors contribuer à ouvrir une piste de recomposition conceptuelle, celle d'un paysage plus discret, où l'invisible et l'imprévisible jouent un rôle essentiel.

Les résultats obtenus ne doivent donc pas être limités à l'intérêt méthodologique et aux constats développés à propos des robiniers mais devraient être vus par le prisme des défis à relever pour les décennies à venir. Incorporer des caractéristiques paysagères dans l'élaboration de la cartographie forestière permet de mieux prendre en compte les dynamiques végétales et donc les phénomènes de conquête ou de repli des peuplements forestiers. De manière encore plus générale, accorder une nouvelle attention aux paysages forestiers, c'est aussi intégrer au champ de recherche du paysage une dimension plus immersive et intégrative : moindre prise de recul pour la vision mais expérience plus directe des lieux. Cette perception de l'espace est nécessairement plus vernaculaire, impliquant moins de discontinuités entre celui qui observe et le lieu qui l'environne. L'enrichissement de la méthode qui consiste à coupler le paysage à la carte permet d'articuler des vecteurs d'accès à l'information proches des perceptions vernaculaires avec les représentations officielles et pourrait conduire les instances de pilotage des politiques forestières à faire évoluer leurs priorités. Aborder les paysages forestiers en transition change donc nos pratiques scientifiques et pourrait bien aussi nous aider à changer le paradigme paysager. Si l'expérience concrète du milieu forestier tempère la prépondérance de la vue sur les autres sensations, elle permet par ce biais le développement de formes d'attachement envers des espaces fermés et peu accessibles qui ont peu à voir avec des notions de pittoresque et de sublime. Ce constat a déjà mené à des réflexions et enquêtes détaillées (Le Floch, 2002; Bigando, 2006), mais il a depuis été exacerbé par la multiplication des enjeux (carbone, biodiversité) et la forte réactivation d'imaginaires, notamment politiques, autour de la forêt (Vidalou, 2017). Une appréhension plus vaste et plus complexe de ces paysages - en l'occurrence forestiers - peut nous aider à relever ces défis, tout en tissant des liens puissants avec notre environnement. 


\section{BIBLIOGRAPHIE}

Balibar, J., « Du paysage représenté au paysage réel », Nouvelle Revue d'esthétique, vol. 22, n², 2008, p. 9-23.

Banos, V. et Dehez, J., « Le bois-énergie dans la tempête, entre innovation et captation ? Les nouvelles ressources de la forêt landaise ", Natures Sciences Sociétés, vol. 25, n 2, 2017, p. 122-133

Bigando, E., «La sensibilité au paysage ordinaire des habitants de la grande périphérie bordelaise (communes du Médoc et de la basse vallée de l'Isle) », thèse de géographie, université Michel de Montaigne - Bordeaux III, 2006.

Couderchet, L., « Le paysage comme paradigme, discussion sur les zonages écologiques et paysagers et leur production », dossier HDR en géographie, université de Bordeaux 3, 2008.

Devanne, A.-S., et Le Floch, S., « L'expérience esthétique de l'environnement : une tension sociopolitique entre l'ordinaire et l'extraordinaire? », Natures Sciences Sociétés, vol. 16, $\mathrm{n}^{\circ} 2,2008$, p. $122-130$.

Fernandez-Carrillo, A., Fuente, D. de la, Rivas-Gonzalez, F. W., Franco-Nieto, A., « A Sentinel-2 unsupervised forest mask for European sites ", Strasbourg, SPIE Remote Sensing, 9-12 septembre 2019.

Gandy, M., « Unintentional landscapes », Landscape Research, vol. 41, n 4, 2016, p. 433-440.

Griselin, M., Nageleisen, S., « "Quantifier” le paysage au long d'un itinéraire à partir d'un échantillonnage photographique au sol », Cybergeo. Revue européenne de géographie, document 253 , janvier 2004, URL : URL : http://journals.openedition.org/cybergeo/3684; DOI : https://doi.org/ $10.4000 /$ cybergeo.3684

Hautdidier, B., Banos, V, Deuffic, P., Sergent, A., « “Leopards” under the pines: an account of continuity and change in the integration of forest land-uses in Landes de Gascogne, France ", Land Use Policy, vol. 79, $\mathrm{n}^{\circ}$ 12, 2018, p. 990-1000.

Institut national de l'information géographique et forestière, Mémento de l'inventaire forestier, édition 2019, URL: https://inventaire-forestier.ign.fr/IMG/pdf/memento_2019_web-2.pdf.

IGN, « BD Forêt(R) Version 2.0. Descriptif de contenu », 2016, URL: https://inventaireforestier.ign.fr/IMG/pdf/dc_bdforet_2-0.pdf

Jenkins, J., «A "deep" aesthetics of contested landscapes: Visions of land use as competing temporalities ", Geoforum, vol. 95, 2018, p. 35-45.

Karasiak, N., Sheeren, D., Fauvel, M., Willm, J., Dejoux, J., Monteil, C., « Mapping tree species of forests in southwest France using Sentinel-2 image time series », " 9th International Workshop on the Analysis of Multitemporal Remote Sensing Images (MultiTemp)», Bruges, Belgique, 27-29 juin 2017.

Kull, C.A., « Forest transitions: a new conceptual scheme », Geographica Helvetica, vol. 72, n 4 , 2017, p. 465-474.

Ladonne, M.-A, « Les Graves de Bordeaux, étude physique et humaine », DES université de Bordeaux III, Institut de géographie, 1946.

Le Floch S., « Les « ramiers « : un espace riverain inaccessible de la Garonne ? », Ethnologie française, vol. 32, 2002/4, p. 719-726. 
Mansfield, B., Biermann, C., Mcsweeney, K., Law, J., Gallemore, C., Horner, L., Munroe, D. K., « Environmental Politics After Nature : Conflicting Socioecological Futures ", Annals of the Association of American Geographers, vol. 105, n² 2, 2014, p. 284-293.

Luginbühl, Y., « Paysage élitaire et paysages ordinaires, Crise du paysage ?” » Ethnologie française, $n^{\circ} 3,1989$, p. 227-238.

Mather, A. S., « The forest transition », Area, vol. 24, n 4, 1992, p. 367-379.

Mather, A. S., Fairbairn, J., Needle, C. L., « The course and drivers of the forest transition: The case of France ", Journal of Rural Studies, vol. 15, nº 1, 1999, p. 65-90.

Nageleisen, S., « Paysages et déplacements, éléments pour une géographie paysagiste », thèse de doctorat de géographie, université de Franche-Comté, 2007, 302 p.

Rebsomen, A., La Garonne et ses affluents de la rive gauche, Bordeaux, Féret et fils éditeurs, 1913.

Sahraoui, Y., Vuidel, G., Foltête, J.-C., Joly, D., « PixScape - un outil logiciel intégré pour l'analyse du paysage visible », Cybergeo. Revue européenne de géographie, document 799, décembre 2016, URL : http://journals.openedition.org/cybergeo/27862; DOI : https://doi.org/10.4000/cybergeo. 27862

Sloan, S., Meyfroidt, P., Rudel, T. K., Bongers, F., Chazdon, R., « The forest transformation: Planted tree cover and regional dynamics of tree gains and losses », Global Environmental Change, vol. 59, article 101988, November 2019.

Vidalou, J.-B., Être forêts. Habiter des territoires en lutte, Paris, La Découverte, 2017.

\section{NOTES}

1. Citant respectivement Rosario Assunto et Yves Lacoste.

2. L'objet forestier invite en effet à une prise en compte de l'expérience concrète et sensorielle d'un paysage, en ce qu'il est parcouru et éprouvé. Pour anecdotique qu'il soit, l'engouement récent en France pour des offres de sylvothérapie basée sur le bain de forêt (shinrin yoku) repose ainsi sur la promesse d'une expérience combinant odorat, ouïe et kinesthésie...

3. Il s'agit du produit Sentinel-2 (ESA) 3A mis à disposition par la plateforme Theia. Il s'agit de synthèses mensuelles de réflectances de surface, dont l'intérêt principal est l'absence de couverture nuageuse.

4. Nous nous sommes basés sur la bibliothèque de machine learning de la suite logicielle OrfeoToolBox. En parallèle à la création d'un premier masquage "forêt " basé sur l'interprétation d'une classification non supervisée (Fernandez-Carrillo et al., 2019), nous avons renseigné une essence dominante pour 385 points de validation «terrain ». Assignant un tiers des points à l'apprentissage, nous avons testé puis sélectionné plusieurs algorithmes de classification, dont les résultats ont été fusionnés selon une règle de majorité.

5. Ayant choisi une nomenclature à sept classes, nous avons retenu trois algorithmes pour construire la classification : Random Forest, puis Support Vector Machine avec noyaux linéaires et polynomiaux.

6. Les correspondances non évidentes entre les mentions « Essence » de la BD Forêt v2 et notre classification sont les suivantes : feuillus, mixte, châtaignier $\rightarrow$ feuillus divers ; pin maritime, pin mélangé, pin autre, conifère $\rightarrow$ chênes décidus; NR (non reconnu) $\rightarrow$ jeunes pins, NC (non cartographié) $\rightarrow$ autres.

7. Avec une valeur de 0,77 pour l'indice Kappa de Cohen, mesuré ici sur l'agrément avec l'ensemble des 314 points du jeu de données « terrain » (apprentissage et validation). 
8. 0,29 pour le Kappa de Cohen, calculé cette-fois ci sur des croisements de superficies.

9. De longue date, l'acacia est reconnu comme partie intégrante des paysages de la région comme le souligne Maire-Anne Ladonne : «La vigne est sans doute la plante caractéristique des Graves. Mais il n'est pas de paysage, même dans la partie la plus vinicole, qui ne soit limité par la ligne sombre des pins ou par un boqueteau plus clair d'acacias. Les taux de boisement, en comparaison de la vigne, sont assez considérables » (Ladonne, 1946, p. 68).

10. Peuplement néanmoins coupé au cours du printemps 2020, pour ne laisser en place que les chênes préexistants.

11. Plan du domaine de Duroy-Suduiraut situé dans les communes de Preignac et Sauternes, Bibliothèque nationale de France, département cartes et plans, GE DL 1848-74-2, 1848, URL: https:// gallica.bnf.fr/ark :/12148/btv1b53087793t

\section{RÉSUMÉS}

Cet article investit la complexité, la diversité des contextes paysagers et des dynamiques du couvert arboré qui leur sont associées. Nous nous appuyons sur l'exemple des espaces boisés dominés par le robinier faux-acacia dans le Sauternais. Introduite en France au XVII ${ }^{e}$ siècle, l'espèce se distingue actuellement par une expansion qui, pour être diffuse et à bas bruit, n'en est pas moins rapide et spectaculaire. Afin d'appréhender la diversité des paysages associés aux robineraies, nous mettons en évidence les formes de disjonctions entre les dynamiques socioécologiques récentes des robineraies et leur reconnaissance officielle via une captation par des méthodes classiques de cartographie et d'inventaire, que ce soit par « le haut « ou « le bas ». Le croisement d'approches proposé (analyse spatiale et enquête photographique, complétées par des entretiens et sources archivistiques) offre une lecture plus fine des modalités de ces transitions forestières et des appréciations contradictoires des paysages associés à "l'acacia ", largement impensées de l'action publique.

This paper investigates the complexity and diversity of landscape contexts and associated tree canopy dynamics. We use the example of wooded areas dominated by the Robinia pseudoacia in the Sauternes region of France. Introduced in France in the $17^{\text {th }}$ century, the expansion of the species, although diffuse and unobtrusive, has nonetheless been rapid and spectacular. In order to apprehend the diversity of landscapes associated with Robinia groves, we highlight the various inconsistencies between the recent socio-ecological dynamics of the groves and their official recognition via classical methods of mapping and listing, either from above or below the forest canopy. The proposed combination of approaches (spatial analyses and photographic surveys, supplemented by interviews and archival sources) offers a more detailed interpretation of the modalities of these forest transitions and the contradictory assessments of the landscapes associated with the "acacia", largely overlooked in public initiatives. 


\section{INDEX}

Mots-clés : transition forestière, trajectoire, visibilité-invisibilité, dynamiques socioécologiques, Robinia pseudoacacia

Keywords : forest transition, trajectory, visibility-invisibility, socio-ecological dynamics, Robinia pseudoacacia

\section{AUTEURS}

\section{SÉBASTIEN NAGELEISEN}

Sébastien Nageleisen est géographe, maître de conférences à l'université Bordeaux Montaigne, membre du laboratoire Passages (UMR 5319 CNRS). Ses axes de recherche sont les paysages (en particulier, les paysages visibles depuis des linéaires) et l'environnement. sebastien.nageleisen[at]u-bordeaux-montaigne[dot]fr

\section{BAPTISTE HAUTDIDIER}

Baptiste Hautdidier est ingénieur-chercheur à l'Inrae, membre de l'unité de recherche Environnement, Territoires et Infrastructures (ETBX). Ses recherches en géographie traitent de la fabrique des découpages de l'espace et de leurs effets sociopolitiques, visant une exigence empirique, technique et critique.

baptiste.hautdidier[at]inrae[dot]fr

\section{LAURENT COUDERCHET}

Laurent Couderchet est géographe, professeur des universités, à l'université Bordeaux Montaigne, membre du laboratoire Passages (UMR 5319 CNRS). Ses domaines de recherche sont la géographie de l'environnement et du paysage, les outils numériques de la géographie. Laurent.Couderchet[at]u-bordeaux-montaigne[dot]fr

\section{ZOÉ GINTER}

Zoé Ginter est géographe, doctorante à l'université Bordeaux Montaigne, à l'Inrae et au laboratoire Passages. Ses domaines de recherches sont la géographie sociale et environnementale, l'économie politique appliquées notamment aux questions forestières. zoe.ginter[at]inrae[dot]fr 